\title{
Guided-mode triggered switching between TE orders of a metal-based grating-waveguide
}

\author{
O. Parriaux \\ parriaux@univ-st-etienne.fr
}

Hubert Curien Laboratory UMR CNRS 5516, University of Lyon, 42000 Saint-Etienne, France

An undulated metal-based dielectric slab waveguide is shown to exhibit a high contrast broadband switching effect in the angular spectrum between the $0^{\text {th }}$ order Fresnel reflection and the propagating $-1^{\text {st }}$ reflected diffraction orders. The switching trigger is the synchronous collinear coupling of an incident TE plane wave to the close-to-cutoff forward- and backward-propagating fundamental $\mathrm{TE}_{0}$ mode of the waveguide via the $+1^{\text {st }}$ and $-2^{\text {nd }}$ order of the periodic undulation.

[DOI: http://dx.doi.org/10.2971/jeos.2015.15040]

Keywords: Optical diffraction, optical gratings, optical switching, optical waveguides

\section{INTRODUCTION}

It is known that a sinusoidal metal grating exhibits a close to $100 \%$ diffraction efficiency under the $-1^{\text {st }}$ order Littrow incidence with a cancellation of the Fresnel reflection at a definite grating depth [1]; the depth at which the $0^{\text {th }}$ order cancellation occurs is smaller for the TM than for the TE polarization. The diffraction efficiency increases monotonically versus the depth, then oscillates periodically with a decrease of the $-1^{\text {st }}$ order efficiency maximum due to increasing metal losses. The angular range of maximum $-1^{\text {st }}$ order around the Littrow angle $\theta_{L}=\sin ^{-1}(\lambda /(2 \Lambda))(\lambda$ is the wavelength in vacuum, $\Lambda$ the grating period) is usually very broad and is limited by the higher reflected orders starting to propagate. It was shown recently [2] that the situation changes radically for the TM polarization if the $-2^{\text {nd }}$ or $+1^{\text {st }}$ order of the grating couples to the forward, resp. backward surface plasmon in which case the efficiency of the propagating $-1^{\text {st }}$ order experiences a sharp drop to 0 at either side of the Littrow angle where the $0^{\text {th }}$ order Fresnel reflection angular spectrum peaks at a close to $100 \%$ maximum [2]. The condition for this high contrast plasmon-mediated switching to take place within a definite angular band around the Littrow angle is $3 K_{g}(=3(2 \pi / \Lambda))$ only slightly larger than twice the plasmon propagation constant $2 \beta=2 n_{e} k_{0}$ ( $n_{e}$ is the surface plasmon effective index and $k_{0}=2 \pi / \lambda$ ); this prevents higher order diffracted waves to propagate. One remarkable feature of this high contrast switching effect in the angular domain between propagating orders is that it is extremely low-loss although mediated by resonant plasmon coupling. The reason for such low-loss plasmonic mechanism was elucidated by a coupledmode analysis [3] which showed that the coupled forwardand backward-propagating plasmon fields re-radiate much before being absorbed into the incident medium along the propagating $0^{\text {th }}$ and $-1^{\text {st }}$ order directions where high contrast constructive or destructive interference takes place depending on the relative phase of the re-radiated fields.

Whether such high contrast triggered switching effect can also exist in the angular spectrum for an incident TE wave is unknown, and difficult to imagine since no surface plasmon can be excited. The present work reports on the search for, and the discovery of a new switching effect for the TE polarization with the mediation of a dielectric-waveguide mode in place of the plasmon mode.

\section{FROM PLASMON- TO WAVEGUIDE-MODE TRIGGER}

Whereas the sinusoidal metallic grating generating the propagating $-1^{\text {st }}$ reflected order inherently also couples the incident wave to the co- and contra-propagating plasmon modes upon an angular tilt in the incidence plane [2], there is no such resonance in the same structure for the TE polarization. Triggering such switching effect for the TE polarization requires the existence of a TE-resonance. This resonance can be a mode of a dielectric layer of refractive index $n_{g}$ and thickness $w$ also coupled by a grating. However, the TE trigger can not consist of a sole dielectric waveguide on a dielectric substrate because there would be propagating orders into the substrate as well since the $0^{\text {th }}$ and $-1^{\text {st }}$ orders propagate in the lower refractive index incident medium (air in the present case). As a result it would be impossible to achieve a high contrast constructive or destructive interference condition in the directions of the $0^{\text {th }}$ and $-1^{\text {st }}$ orders in the cover medium unlike in the plasmonic case where the sole $0^{\text {th }}$ and $-1^{\text {st }}$ orders propagate [3]. Consequently, the dielectric waveguiding layer must have a metallic substrate very much like the plasmonic structure as illustrated in Figure 1.

In analogy to the plasmon-triggered TM switching [2], the plasmon mode is now replaced by the fundamental $\mathrm{TE}_{0}$ mode which the grating couples either in the backward direction via its $-2^{\text {nd }}$ order when the incidence angle $\theta_{-2}$ is smaller than the $-1^{\text {st }}$ order Littrow angle or via its $+1^{\text {st }}$ order when $\theta_{1}$ is larger 


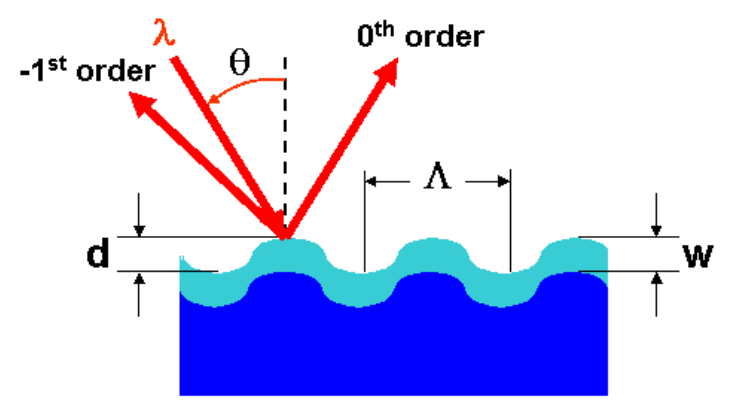

FIG. 1 Dielectric waveguiding layer of index $n_{g}$ and thickness $w$ conformally deposited on a sinusoidal metal grating of period $\Lambda$ and depth $d$ with TE incidence at wavelength $\lambda$ under the angle $\theta$ and the sole $0^{\text {th }}$ and $-1^{\text {st }}$ reflected orders propagating in the incidence medium.

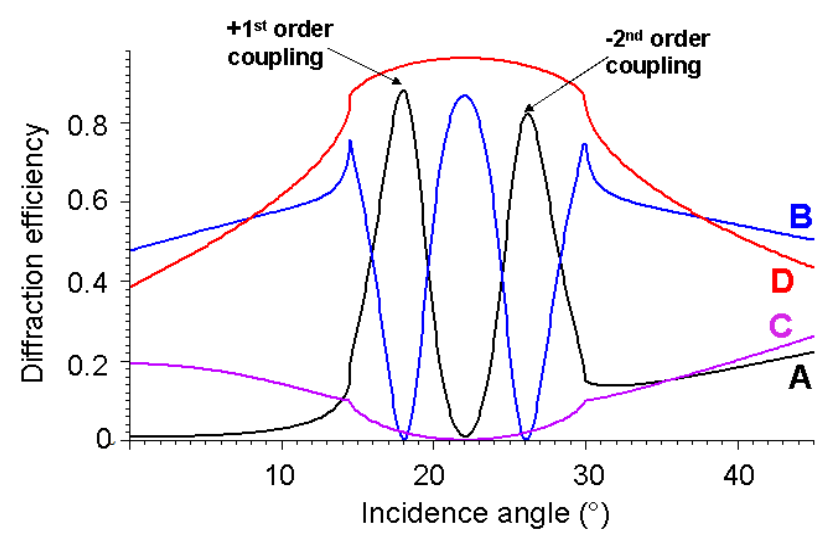

FIG. $2-1^{\text {st }}$ and $0^{\text {th }}$ order angular spectra summarizing the design process of the $\mathrm{TE}_{0}$ waveguide-mode-triggered switch, $\lambda=633 \mathrm{~nm}, \Lambda=844 \mathrm{~nm}$, undulated silver substrate of complex permittivity $\epsilon_{m}=-16.1+i 1.1$. Curves $C$ and $D$ : optimized sinusoidal grating depth $d=320 \mathrm{~nm}$ in a silver substrate without dielectric layer achieving maximum $-1^{\mathrm{st}}$ order diffraction efficiency and cancellation of the $0^{\text {th }}$ order under the Littrow mount at 22 degree incidence. Curves A and B: optimized grating depth $d=240 \mathrm{~nm}$ and waveguide thickness $w=180 \mathrm{~nm}\left(n_{g}=1.46\right)$ achieving high contrast $0^{\text {th }}$ to $-1^{\text {st }}$ order switching with cancellation of the $-1^{\text {st }}$ order at the mode coupling angles.

than the Littrow angle $\theta_{L}$. The corresponding phase matching conditions are, respectively:

$$
k_{0} \sin \theta_{-2}+k_{0} n_{e}=2 K_{g}
$$

and

$$
k_{0} \sin \theta_{1}+K_{g}=k_{0} n_{e}
$$

where $K_{g}$ is the grating constant $2 \pi / \Lambda$ corresponding to the Littrow angle $\theta_{L}$ at the wavelength $\lambda$.

\section{GENERAL DESIGN AND PROPERTIES}

The design process starts by finding numerically the depth of a sinusoidal grating at a silver surface at which the TE $0^{\text {th }}$ order cancels and the $-1^{\text {st }}$ order is maximum under the Littrow condition. The considered optogeometrical parameters are the same as those of the plasmonic structure [2] to permit a comparison of the optical function: 22 degree incidence and $\lambda / \Lambda=633 / 844 \mathrm{~nm} / \mathrm{nm}=0.75$ with air overlay in the absence of dielectric layer; the metal is silver too. Curves $C$ and D in Figure 2 represent the $0^{\text {th }}$ and $-1^{\text {st }}$ order spectra obtained with a grating depth $d=320 \mathrm{~nm}$; the two kinks at 14.5 and 30 degrees on these two smooth curves represent the cutoffs of the

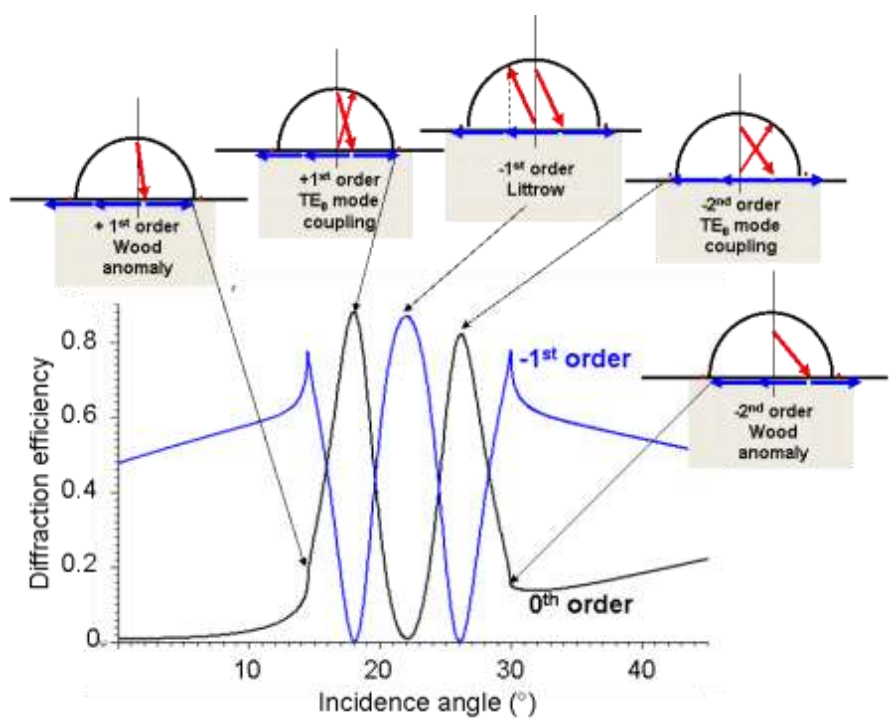

FIC. 3 Waveguide-mode triggered switching between the $0^{\text {th }}$ and $-1^{\text {st }}$ TE orders in the angular spectrum centered on the $-1^{\text {st }}$ order Littrow mount at 22 degree incidence with all features illustrated in the Ewald circles in which the blue vectors represent the grating constant $K_{g}$, the red spots the propagation constant $\beta$ of the waveguide mode, and the red arrows the wave vectors of the incident and $0^{\text {th }}$ and $-1^{\text {st }}$ order diffracted waves. The considered optogeometrical parameters are those of Figure 2, curves $A$ and $B$.

$+1^{\text {st }}$ and $-2^{\text {nd }}$ diffraction orders respectively. The sinusoidal metal undulation is now coated with a conformal dielectric layer; similarly to the plasmon-triggered structure $[2,3]$ the grating depth is then varied to find out whether a cancellation of the $-1^{\text {st }}$ order amplitude at either side of, and close to the Littrow angle can also be obtained; the $-1^{\text {st }}$ and $0^{\text {th }}$ order spectra B and A of Figure 2 show that it is the case indeed: a grating depth of $240 \mathrm{~nm}$ for a $180 \mathrm{~nm}$ thick dielectric layer of 1.46 index $n_{g}$ do lead to a cancellation of the $-1^{\text {st }}$ order and a peak of the Fresnel reflection.

It is at the angles $\theta_{-2}$ and $\theta_{1}$ of synchronous $\mathrm{TE}_{0}$ mode coupling as given by expressions (1) and (2) that the $0^{\text {th }}$ order Fresnel reflection reaches its maximum, and zero at the Littrow angle $\theta_{L}$, once the grating depth is adjusted so as to give rise to constructive or destructive interference in the incident medium (here air) in the directions of the $0^{\text {th }}$ and $-1^{\text {st }}$ orders. For the diffraction process to involve the $-2^{\text {nd }}$, $-1^{\text {st }}, 0^{\text {th }}$ and $+1^{\text {st }}$ orders only, and to have them to operate within the angular width of the $-1^{\text {st }}$ order Littrow condition $2 \lambda / \Lambda-1<\sin \theta<1-\lambda / \Lambda$ between the cutoffs of the $-2^{\text {nd }}$ and $+1^{\text {st }}$ orders, the propagation constant $\beta$ of the $\mathrm{TE}_{0}$ mode must be very small, i.e., the mode must be close to its cutoff ( $n_{e}$ close to 1 in the considered case of air incident medium whatever the refractive index $n_{g}$ of the dielectric waveguide layer).

Figure 3 represents synthetically in the reciprocal space the relationship between all involved spatial frequency vectors in the Ewald circle at the specific features of the angular spectra. The considered structure is that leading to the $-1^{\text {st }}$ and $0^{\text {th }}$ order spectra B and A of Figure 2.

Unlike in the TM case where the plasmon effective index is essentially determined by the metal permittivity [2] - and to a 


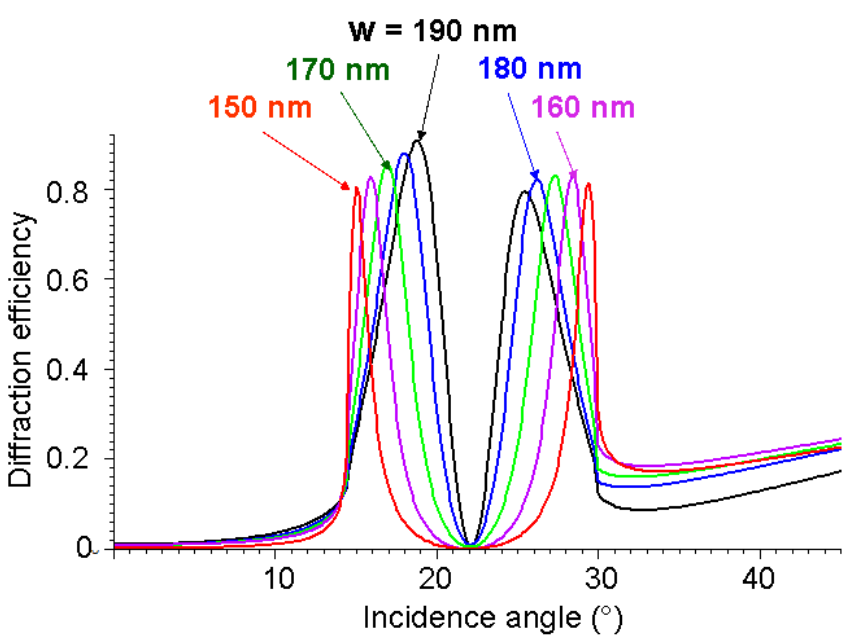

FIG. 4 Tailoring of the angular spectral width of the $0^{\text {th }}$ to $-1^{\text {st }}$ order switching by changing the thickness of the dielectric layer of refractive index $n_{g}=1.46$ with $w$ as a parameter. The $o^{\text {th }}$ order spectrum only is represented. The corresponding effective index of the $\mathrm{TE}_{0}$ mode with increasing thickness is 1.010, 1.024, 1.042, 1.06, 1.072 .

much lesser extent by the grating depth - the effective index of the $\mathrm{TE}_{0}$ mode of the dielectric layer is strongly dependent of the dielectric layer thickness $w$. This means, as illustrated in Figure 4 , that the angular distance between $0^{\text {th }}$ and $-1^{\text {st }}$ order maxima can be adjusted by the layer thickness $w$. It is noteworthy that all 5 spectra have quasi-zero $0^{\text {th }}$ order at the Littrow angle for the same grating depth of $240 \mathrm{~nm}$. Worth noting also is the fact that the absorption loss (between 10 and $20 \%$ as the complement to 1 of the diffraction efficiency maxima) is comparable with the absorption loss of the plasmon-triggered switch [2].

Setting the incidence angles $\theta_{1}$ and $\theta_{-2}$ within the Littrow angular width at which the maxima of the $0^{\text {th }}$ order is desired determines the needed effective index $n_{e}$ from the conditions in Eq. (1) or Eq. (2). Inserting the desired ne into the dispersion equation of a dielectric slab waveguide $\mathrm{TE}_{0}$ mode [4] with air superstrate and metal substrate of real part of negative permittivity $\epsilon_{m r}$ yields the thickness $w$ of the needed waveguide layer:

$$
w=\frac{\left(\tan ^{-1}\left(\sqrt{\frac{n_{e}^{2}-\epsilon_{m r}}{n_{g}^{2}-n_{e}^{2}}}\right)+\tan ^{-1}\left(\sqrt{\frac{n_{e}^{2}-1}{n_{g}^{2}-n_{e}^{2}}}\right)\right)}{k_{0} \sqrt{n_{g}^{2}-n_{e}^{2}}}
$$

Introducing $w$ obtained from Eq. (3) into an exact code calculating the diffraction of the undulated metal substrate loaded with a dielectric layer of uniform thickness $w$ using the Chandezon method [5] will however not give the $0^{\text {th }}$ order maximum exactly where it was desired at $\theta_{1}$ and $\theta_{-2}$ because dispersion equation (Eq. (3)) is for a planar metal-based waveguide structure without undulation. The fact that the dielectric waveguide is undulated decreases slightly the effective index; however, the provided $w$ is a safe departure point for a numerical optimization. Besides, under the hypothesis of a close-to-cutoff $\mathrm{TE}_{0}$ mode, the transcendental dispersion equation (Eq. (3)) can be explicitly solved for the effective index $n_{e}$ after some algebraic approximations using the fact that $n_{e}$ is very close to 1 :

$$
n_{e}=1+\delta
$$

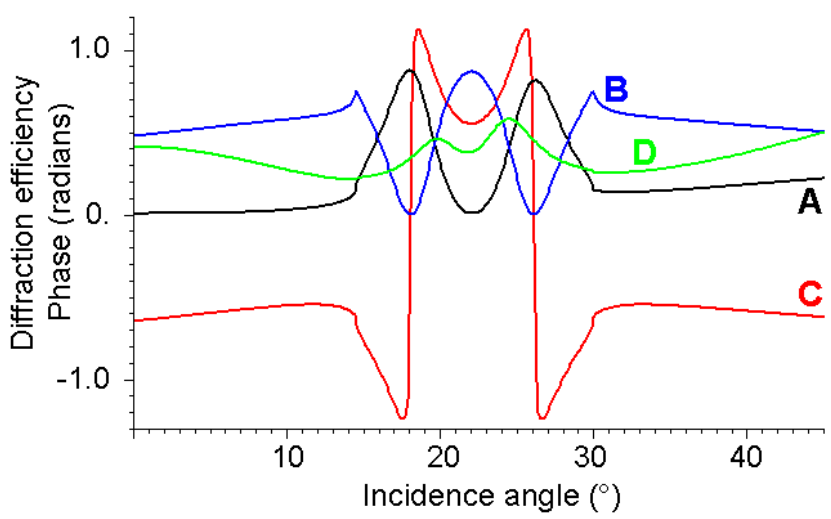

FIG. 5 Phase spectrum in radians of the fields of the $-1^{\text {st }}$ order (curve C) corresponding to the structure of Figure 2 (curves $A$ and $B$ ). The green curve $D$ is the $0^{\text {th }}$ order spectrum of the TM polarization in the same structure.

where

$$
\delta=\frac{n_{g}^{2}-1}{2}\left[\frac{\pi}{2}-\sqrt{n_{g}^{2}-1}\left(k_{0} w+\frac{1}{\sqrt{1-\epsilon_{m r}}}\right)\right]^{2}
$$

The evidence that the cancellation of the $-1^{\text {st }}$ order and maxima of the $0^{\text {th }}$ order occur under the condition of a TE mode excitation is given in Figure 5 which shows, in addition to the $0^{\text {th }}$ and $-1^{\text {st }}$ order spectra used in Figure 2, the phase profile of the $-1^{\text {st }}$ order field: there is a large and sudden phase jump of 2.4 radians characteristic of mode excitation at the angular position of the two $0^{\text {th }}$ order maxima.

Interestingly, calculating the TM angular spectrum of the $0^{\text {th }}$ order (curve D) of the very same structure (curves A and B) shows that obviously the contrast is poor since the structure is not designed for the plasmon-triggered switching, but reveals that the $0^{\text {th }}$ order maxima are notably closer to each other than in the TE spectra which means that the effective index of the $\mathrm{TM}_{0}$ mode is larger than the $\mathrm{TE}_{0}$ 's; the reason for this lies on the fact that the substrate is metallic, therefore the fundamental mode is of plasmonic nature (the field in the dielectric waveguide is evanescent) and its effective index is as large as 1.51 .

An important comment must be made at this stage about the phenomenology of the designed waveguide-mode mediated switching effect: the coupled-mode interpretation of the TM plasmon-mediated switch [3] reveals that the astonishingly low-loss plasmonic effect and its high interference contrast originate in the plasmon radiation strength of the rather deep grating being notably larger than the absorption coefficient of the plasmon mode. Although the coupled-mode analysis of the present TE switch hasn't been developed, the same rationale can be applied: the $\mathrm{TE}_{0}$ waveguide mode coupled by the $+1^{\text {st }}$ and $-2^{\text {nd }}$ orders of the grating is radiated back to the incident medium along the $0^{\text {th }}$ and $-1^{\text {st }}$ order directions before being absorbed by the metallic substrate. A sign of this is the fact that the direct $-1^{\text {st }}$ order excitation of the $\mathrm{TE}_{0}$ mode in the same structure exhibits a loss of about $70 \%$ (99\% at a grating depth of $50 \mathrm{~nm}$ ) whereas the loss associated with the $+1^{\text {st }}$ and $-2^{\text {nd }}$ order coupling (see Figure 2 ) is about 15 and $20 \%$ only.

The design process and all examples above use as a model a dielectric layer of 1.46 refractive index $n_{g}$. This is by no means 


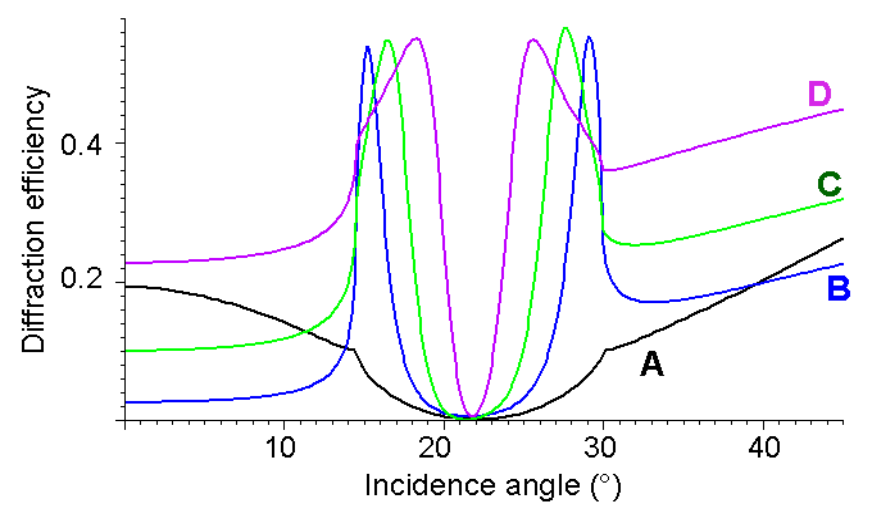

FIG. 6 Reflected $0^{\text {th }}$ order TE angular spectra with $\Lambda=844 \mathrm{~nm}, \lambda=633 \mathrm{~nm}, n_{g}=2.4$. A: No dielectric layer, $d=320 \mathrm{~nm}$. B: $w=60 \mathrm{~nm}, d=230 \mathrm{~nm}, n_{e}=1.077$. C: $w=62 \mathrm{~nm}$, $d=200 \mathrm{~nm}, n_{e}=1.100$. D: $w=64 \mathrm{~nm}, d=170 \mathrm{~nm}, n_{e}=1.130$.

a restriction since it is the effective index of the $\mathrm{TE}_{0}$ mode that essentially matters. As an evidence of this, Figure 6 is the analogue of Figure 4 with a waveguide layer of refractive index $n_{g}=2.4$ which may approximately correspond to a $\mathrm{ZnS}, \mathrm{TiO}_{2}$ or diamond layer in the visible and near infrared domains. In the presence of a high index layer the waveguide thickness $w$ must be adjusted (i.e., decreased) to give rise to an effective index $n_{e}$ of the $\mathrm{TE}_{0}$ mode sufficiently close to its cutoff (i.e., close to 1 here). The angular spectra (the $0^{\text {th }}$ order Fresnel reflection only is shown in Figure 6) exhibit the same general pattern, but evidently the control over the layer thickness $w$ is much more critical to give rise to a sufficiently small effective index. Curves B, C and D of Figure 6 show here too, as in Figure 4, a feature which the plasmon-triggered switch does not exhibit: changing the waveguide thickness $w$ permits to vary the angular distance between $0^{\text {th }}$ and $-1^{\text {st }}$ order maxima/minima, i.e., the operation range of the switch. Using a high index dielectric layer is however not so interesting practically since the $0^{\text {th }}$ order remains large outside the angular switching range and the absorption loss is slightly larger.

\section{CONCLUSION}

The present analysis shows that the plasmon-triggered switching effect between two free-space orders first reported in [2] and elucidated in [3] can be extended to the TE polarization by providing a resonance in the form of the fundamental $\mathrm{TE}_{0}$ guided mode of a close-to-cutoff thin dielectric layer deposited onto a metal grating. Similarly to the plasmonmediated case, the TE switch exhibits absorption losses which are notably smaller than under direct mode coupling by the same grating. An interesting, although unexpected, common characteristic of the plasmon and dielectric mode-triggered structures lies in that the very same metal grating depth of $240 \mathrm{~nm}$ gives rise to the TM switching with air in the grooves and to the TE switching with a 150-190 nm thick dielectric layer of index 1.46 covering the sinusoidal corrugation. The specific interest of the TE switch is to enable the angular distance between $0^{\text {th }}$ order maxima to be adjusted by controlling the waveguide thickness. The present analysis was made assuming a conformal dielectric film deposited onto an undulated metal substrate; this condition would not be fulfilled with a spin-coated dielectric film (polymer or Sol-Gel waveguide), that is why vacuum deposition technologies like PVD Physical Vapour Deposition, (PE)CVD (Plasma Enhanced) Chemical Vapour Deposition, or ALD Atomic Layer Deposition should be used to satisfy this assumption.

This surface-wave-triggered switching effect can be used in the field of security and anti-counterfeit applications where angular tilting is often used. It could also be used to separate or combine two broad wavelength spectra at fixed incidence angle by exploiting the waveguide dispersion characteristics.

\section{References}

[1] E. Popov, L. Tsonev, and D. Maystre, "Gratings - General properties of the Littrow mounting and energy flow distribution," J. Modern Optics 37, 367-377 (1990).

[2] J. Sauvage-Vincent, Y. Jourlin, V. Petiton, A. V. Tishchenko, I. Verrier, and 0. Parriaux, "Low-loss plasmon-triggered switching between reflected free-space diffraction orders," Opt. Express 22, 13314-13321 (2014).

[3] A. V. Tishchenko, and 0. Parriaux, "Coupled-mode analysis of the low-loss plasmon-triggered switching between the $0^{\text {th }}$ and $-1^{\text {st }}$ orders of a metal grating," IEEE Photon. J. (2015) article in press.

[4] P. K. Thien, "Light waves in thin films and integrated optics," Appl. Optics 10, 2395-2413 (1971).

[5] N. Lyndin, MC Grating Software, http://www.mcgrating.com/ 